Hydrology and Earth System Sciences, 3(1), 15-30 (1999) (C) EGS

\title{
Combined modelling of shortwave and thermal radiation for one-dimensional SVATs
}

\author{
D. Pearson, ${ }^{1}$ C.C. Daamen, ${ }^{2}$ R.J. Gurney ${ }^{1}$ and L.P. Simmonds ${ }^{3}$ \\ ${ }^{1}$ Environmental Systems Science Centre, Harry Pitt Building, Whiteknights, PO Box 238, Reading RG6 6AL, UK. \\ 2 Kerikeri Research Centre, PO Box 23, Kerikeri, Bay of Islands, New Zealand. \\ 3 Department of Soil Science, University of Reading, Whiteknights, PO Box 233, Reading RG6 6DW, UK.
}

\begin{abstract}
Expressions for the upwelling and downwelling fluxes of optical and thermal radiation between soil, vegetation and the sky are derived, under certain simple assumptions. These are that interception of radiation by the vegetation is a purely geometric effect, while scattering is isotropic, with a strength given by a single-scattering albedo in the optical part of the spectrum, and by Kirchoff's Law in the thermal. The soil is assumed to be a lambertian reflector, also scattering according to an albedo and Kirchoff's Law. The model, called RM, conserves energy exactly. As part of a SVAT, it is driven by measured insolation instead of net radiation, with little increase in computational cost and number of parameters.
\end{abstract}

\section{Introduction}

During the past decade, many SVAT models have incorporated explicit consideration of the interactions between multiple sources of water for evaporation (e.g. soil and canopy surfaces) when describing land surface/atmosphere exchanges. Many of these have been based on extensions of the Penman-Monteith equation proposed by Shuttleworth and Wallace (1985) and Choudhury and Monteith (1988); these attempt to take account of interactions between the contributing evaporation surfaces (e.g. soil and canopy surfaces) that arise when the aerodynamic transfers of sensible and latent heat from the individual surfaces interact within the canopy air space. Much attention has been given to the parameterisation of the various surface, aerodynamic and boundary layer resistances that describe the flow paths of sensible and latent heat from the individual surfaces to the point at which the flows merge, and then the combined flow path from the within-canopy air space to some reference height in the atmosphere above the canopy.

The exchanges of sensible and latent heat are driven by solar forcing, expressed in the Shuttleworth and Wallace (S-W) and in the Choudhury and Monteith (C-M) evaporation equations in terms of the net radiation allocated to each contributing surface, where the net radiation $\left(R_{n}\right)$ is the difference between the total downwelling radiative flux (including shortwave and thermal) and total upwelling radiative flux. However, interactions between solar radia- tion and complex bodies such as soil surfaces covered by a sparse vegetation canopy are difficult to measure and to model; a full mathematical description of solar forcing within a SVAT appropriate for sparsely vegetated land would be very difficult to apply, requiring a large number of parameters that are difficult to measure and rarely available. Nevertheless, the problems should not be dismissed because the sensible and latent heat fluxes can be quite sensitive to radiative forcing. For example, HendersonSellers et al. (1993) note that changes of 0.02 or more in albedo can result in substantial changes in modelled surface fluxes and surface temperature.

Various formulations of radiative forcing have been suggested and implemented. Where accuracy is not the foremost requirement, or is allowed to be degraded in the trade off between computational complexity and fast calculation, simple formulae have been used. For example, Franks et al. (1997) assumed a Penman-Monteith formulation, without modelling radiative transfer at all; nevertheless, this was sufficiently accurate for their purpose. Some more complex models use the methods of radiative transfer to simulate the transport of sunlight in the canopy. This degree of complexity is required for many purposes when there are multiple sources and sinks of radiation and other fluxes, as in two-source models such as that discussed in detail in the rest of this paper. For example, Hope et al. (1986) used the TERGRA SVAT, and incorporated Verhoef's (1984) SAIL, a simple radiative 
transfer model; $\mathrm{SiB}$ [Sellers et al. (1986)] used a related but much more complicated method, simplified to some extent by Xue et al. (1991) in the SSiB model. MAESTRO [Wang and Jarvis (1990)], a SVAT which also models transport of carbon, used an unusually comprehensive model of the radiative properties of the canopy, in which crowns are considered as assemblages of interacting elements.

Finally, the most complex radiation transport models (e.g. Gerstl and Borel (1992), Pearson (1997)), which accurately simulate the directional reflectance of canopies, are usually not applicable for inclusion in SVATs because of their complexity, although they are useful for reference. For most purposes, a balance between the degrees of complexity of the different compartments or modules of a SVAT is desirable.

Although there has been some use of the more complex models, the requirement for additional observations has limited their use. Many are also not fully coupled to a SVAT. In the context of modelling canopies using the dual-source combination equation approach (i.e. variants of the S-W or C-M Equations), the most widely adopted approach to modelling net radiation and its partitioning is still to start with some estimation of the overall net radiation available, and then to partition $R_{n}$ between that available to the soil $\left(R_{s}\right)$ and canopy $\left(R_{v}\right)$ surfaces. Many SVATs [e.g. Choudhury and Monteith (1988), Daamen $(1993,1997)]$ assume that the partitioning of net radiation can be described using Beer's law for exponential attenuation and assume that the appropriate attenuation coefficient $(\alpha)$ is that which describes the attenuation of downwelling solar radiation. This gives rise to the following simple equations:

$$
\begin{aligned}
& R_{s}=R_{n} \exp \left(-\alpha A_{L}\right), \\
& R_{v}=R_{n}-R_{s}=R_{n}\left[1-\exp \left(-\alpha A_{L}\right)\right],
\end{aligned}
$$

where $R_{s}$ is the net radiation available to the soil, $R_{v}$ is the net radiation available to the vegetation, $\alpha$ is an attenuation coefficient and $A_{L}$ is the leaf area index. Wallace $e t a l$. (1986) find that $\alpha=0.41$ for West African millet crops, while Choudhury et al. (1987) find $\alpha=0.5$ for wheat.

This approach is usually implemented assuming a constant attenuation coefficient (and hence constant net radiation partitioning) throughout the day, whereas in reality, the attenuation depends on the obliquity of the path through the canopy.

Accurate partitioning of net radiation might be crucial in accurate simulation of hourly or daily surface fluxes, because the fractions of net radiation that are dissipated as sensible and latent heat can be very different for the soil and canopy surfaces, and have very different diurnal patterns. For example, in the case of a sparse millet crop on a sandy soil in Niger it is typical for the majority of net radiation allocated to the canopy to be dissipated as latent heat (via transpiration) whereas evaporation from the soil surface will rise to a sharp peak by mid-morning before decreasing sharply once the few millimetres of soil closest to the surface dry out. In such circumstances, overallocation of net radiation to the soil would be expected to result in underestimation of total evaporation.

A further objection to the use of Eqns. 1 and 2 is that they treat the shortwave and thermal components of net radiation together. However, in reality these two wavebands are subject to different transport laws.

This paper presents a new formulation for the radiative transfers within a SVAT that is more realistic than Beer's law without being too complex, which is physically reasonable, and which can be driven from knowledge of downwelling solar radiation (this is often the only radiation flux of which measurements are readily available). The objective is to produce a model that requires no further information about the size and architecture of the canopy beyond the leaf area index and attenuation coefficient for downwelling solar radiation that is already required by approaches based on Beer's law. This is done by analysis of the detailed energy balance between the soil, vegetation and atmosphere in a simplified representation of the canopy. The approach builds on ideas in the radiation module of the SiSPAT SVAT [SiSPAT is discussed in detail by Braud et al. (1995); it uses a radiation model proposed by Taconet et al. (1986)], following the multiple reflections of shortwave and thermal radiation between the foliage and the soil. In the present study, the calculation is extended by more accurate representation of the radiative scattering processes in the foliage, and by allowing diurnal variation of these processes: it is also indicated how to extend the method to more complex radiative transfer methods, should these be required.

Reflected solar flux and net radiation can be calculated as diagnostics of correct functioning of the new model, which will be referred to as RM. It is incorporated into an existing SVAT model-SWEAT-[Daamen (1993, 1997)] that is based on the Shuttleworth-Wallace two-source approach, to create a new model (SWEAT2). The paper explores how the partitioning of net radiation between $R_{v}$ and $R_{\mathrm{s}}$ using RM differs from that using a simple Beer's law approach, and the implications for the prediction of surface temperature.

The new radiation model is a significant advance on other single-layer models. Although the derivation is lengthy, the end result is not complex and is quick to run on a computer.

\section{The models}

SOIL WATER, ENERGY AND TRANSPIRATIONSWEAT

The work to be discussed was applied as an upgrade to the SWEAT model, an overview of which is presented here. 
Daamen $(1993,1997)$ provides further details. SWEAT is a one-dimensional model which simulates the movement of water and heat in the soil profile above a nominated depth (e.g. $2 \mathrm{~m}$ ), up to a reference height in the air above the vegetation (e.g. at a height of $5 \mathrm{~m}$ ). In the soil, fluxes of liquid water, water vapour and sensible heat are simulated using Richards' equation, thermally enhanced Fickian diffusion of vapour and the Philip and de Vries (1957) approach to modelling heat flow. Following Campbell (1985), the coupled partial differential equations are solved by a fully implicit backward-difference scheme in time, approximately centred in the spatial grid. This numerical scheme can also be considered as a resistancecapacitance network. Spatial centering is approximate because the spatial grid's spacing increases with depth. This enables efficient calculation of transport of water close to the soil's surface, where changes are rapid because of fluctuations in forcing at the boundary. The equations are non-linear, so a Newton-Raphson method is used to solve the implicit set of equations at each time step.

In the canopy air space, fluxes of water vapour and sensible heat from the vegetation and soil are described using a two-source method developed from that of Shuttleworth and Wallace (1985). The vegetation is assumed to have a stomatal resistance $r_{s t}$ in series with a boundary layer resistance $r_{b l}$. The latter terminates in the air space within the canopy, which is connected by a further resistance $r_{\text {scan }}$ to the surface of the soil and by another resistance $r_{a}$ to the atmosphere at the reference height. Thus, the vegetation and the soil can interact within the canopy's air spacethis is an important consideration in the sparse crops for which SWEAT was designed, where evaporation from the leaves can be increased by heating by hot air rising from the soil between the plants, or reduced by humidification of the within-canopy air space following evaporation from the soil's surface. Methods of calculating the resistances are taken from recently published literature. The movement of water from the bulk soil to the site of evaporation within leaves is modelled using an Ohm's law analogue of water flow in the soil-plant system, incorporating radial root and soil resistances (both of which depend on rootlength density) in series with the hydraulic resistance in the shoot. The linkage between stomatal resistance and soil water is via leaf water potential, there being a threshold value of the potential below which stomatal resistance increases dramatically.

The model is forced by meteorological data at the reference height (temperature, net radiation, relative humidity, wind speed and rainfall) and by an assumption of unit hydraulic gradient (i.e. steady-state gravity-driven flow with homogeneous matric potential) at the nominated depth in the soil that is greater than the depths of interest. Net radiation is required as an input, and is partitioned between the soil and vegetation according to Eqns. 1 and 2.
THE RADIATION MODEL-RM

\section{Introduction}

In the models vegetation moderates the supply of energy to the soil and canopy surfaces, and allows subsurface water to enter the atmosphere by transpiration. In RM, the physics of photosynthetically active radiation is not treated differently from that of the rest of the visible/NIR spectrum, while transfer of thermal radiation is considered as a different phenomenon. Hence two broad wavebandsshortwave (optical) and longwave (thermal)-have been considered. Thermal radiation is assumed to arise isotropically from the surface of the soil, isotropically from the vegetation, and isotropically from the sky; shortwave radiation is incident isotropically from the sky, and as a delta-function in direction from the sun. Various approximations are already evident: isotropy, a delta-function, and the simple division between shortwave and thermal radiation. In developing $\mathrm{RM}$, horizontal homogeneity of all physical quantities is assumed, as is assumed also in the SWEAT model.

Interception of radiation by the vegetation is taken to be purely a geometric effect, i.e. it is assumed that a beam of radiation (shortwave or thermal) is attenuated by striking the physical elements of the vegetation. Intercepted radiation is assumed to be absorbed or scattered. The scattered component is scattered with equal isotropic fluxes upward out of the top of the vegetation, and downward from the bottom. That is, the physical depth of the vegetation is ignored, but it is assumed to intercept a portion of radiation incident on it; of this, some is absorbed and the rest scattered isotropically into the exitant hemispheres above and below. However, the fate of intercepted radiation differs between shortwave and thermal. If a flux of shortwave radiation is intercepted, a fraction is scattered given by the single-scattering albedo $a_{v}$. In the thermal spectrum, it is assumed (as an approximation) that Kirchoff's law applies to the whole thermal waveband, i.e. the emissivity of the vegetation, $\epsilon_{v}$, is equal to its absorptivity, $a_{v}$. This implies that the vegetation has a single-scattering albedo of $1-\epsilon_{v}$ in the thermal waveband. It is not usual in simple thermal models to take account of scattered thermal radiation. Indeed, given the many approximations involved in the model, and that typically $0.95<\epsilon_{v}<0.99$ [Monteith and Unsworth (1990)], the flux of scattered thermal radiation can probably be ignored as a reasonable approximation. However, it is taken into account in RM to conserve energy exactly in the model. This is possible because the adding method used is efficient. It is assumed that intercepted thermal flux is scattered equally and isotropically upwards and downwards, exactly as the shortwave flux.

The assumed isotropy is not physically realistic, because the light scattered by a canopy is generally rather anisotropic, as discussed by Gerstl and Borel (1992) or Pearson (1997). However, in the present context, only fluxes of radiation are of interest and these are calculated 
by integrating the radiance (with a geometric factor) over a hemisphere of exitance, thus erasing information about anisotropy. So, the approximation is appropriate in this context.

The soil is assumed to be a Lambertian reflector (see Appendix A) with an albedo $a_{s}$ in the shortwave and an albedo $1-\epsilon_{s}$ in the thermal infrared, where $\epsilon_{s}$ is the emissivity of the soil.

\section{An adding method}

All relevant thermal and shortwave fluxes are calculated by use of an adding method. This is a formalism by which all orders of multiple scattering are implicitly and exactly (within the assumptions of the model) taken into account. A clear treatment of adding methods for scattering media is given by Grant and Hunt (1969a, 1969b); it is a standard analytical tool in radiative transfer theory. However, the optical addition of a substrate to a scattering medium is not widely understood, so it is derived here.

In Fig. 1(a), consider a horizontally homogeneous field of radiation quantified as $\bar{D}$, incident on the surface of a scattering medium. $\bar{D}$ can be a directional radiance or a hemispherically integrated flux, as the notation to be developed applies to both. Now, the medium transmits a certain portion of $\bar{D}$, given by a transmission operator $\hat{T}$, and reflects an amount given by a reflection operator $\hat{R}$. Let the substrate reflect a portion of the radiation incident upon it, given by a reflection operator $\hat{B}$. If the radiative quantities in question are directional radiances, these operators are integral transforms (e.g. $\hat{B}$ could represent integration of the radiance multiplied by the bidirectional reflectance distribution function); if fluxes, they are just coefficients; if radiances discretized by a discrete ordinates method, they are transfer matrices. Henceforth, it is assumed that they are fluxes.

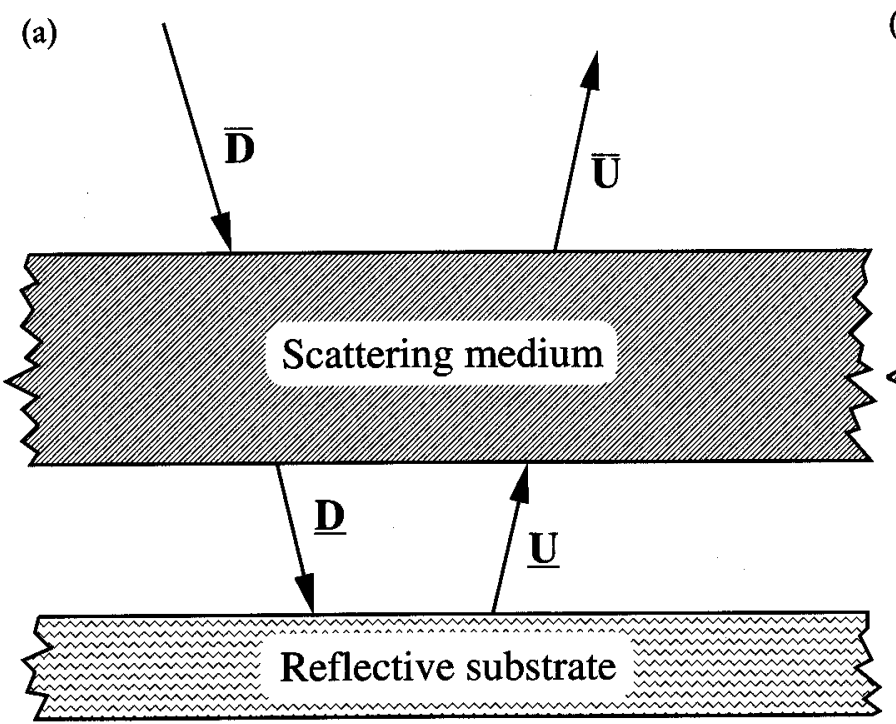

(b)

$$
\underline{U}=\hat{B} \underline{D} .
$$

If the flux $\bar{D}$ incident on top of the vegetation is given, then equations 3-5 are three simultaneous linear equations in the three unknown quantities $\underline{D}, \underline{U}$ and $\bar{U}$.

Elimination of $\underline{U}$ between equations 4 and 5 yields $\underline{D}=\hat{T} \bar{D}+\hat{R} \hat{B} \underline{D}$, from which the following solution follows by rearrangement:

$$
\underline{D}=[\hat{I}-\hat{R} \hat{B}]^{-1} \hat{T} \bar{D}
$$

where $\hat{I}$ is the identity operator.

Then Eqn. 5 gives $\underline{U}$; and then Eqn. 3 gives $\bar{U}$. Thus, all relevant radiative quantities are obtained from knowledge of the incident radiation and the transfer operators.

Equation 6 can be understood by expanding $[\hat{I}-\hat{R} \hat{B}]^{-1}$ in a binomial series:

$$
\begin{aligned}
\underline{D} & =\left[\hat{I}-\hat{R} \hat{B}+(\hat{R} \hat{B})^{2}+(\hat{R} \hat{B})^{3}+\ldots\right] \hat{T} \bar{D} \\
& =\hat{T} \bar{D}+\hat{R} \hat{B} \hat{T} \bar{D}+\hat{R} \hat{B} \hat{R} \hat{B} \hat{T} \bar{D}+\hat{R} \hat{B} \hat{R} \hat{B} \hat{R} \hat{B} \hat{T} \bar{D}+
\end{aligned}
$$

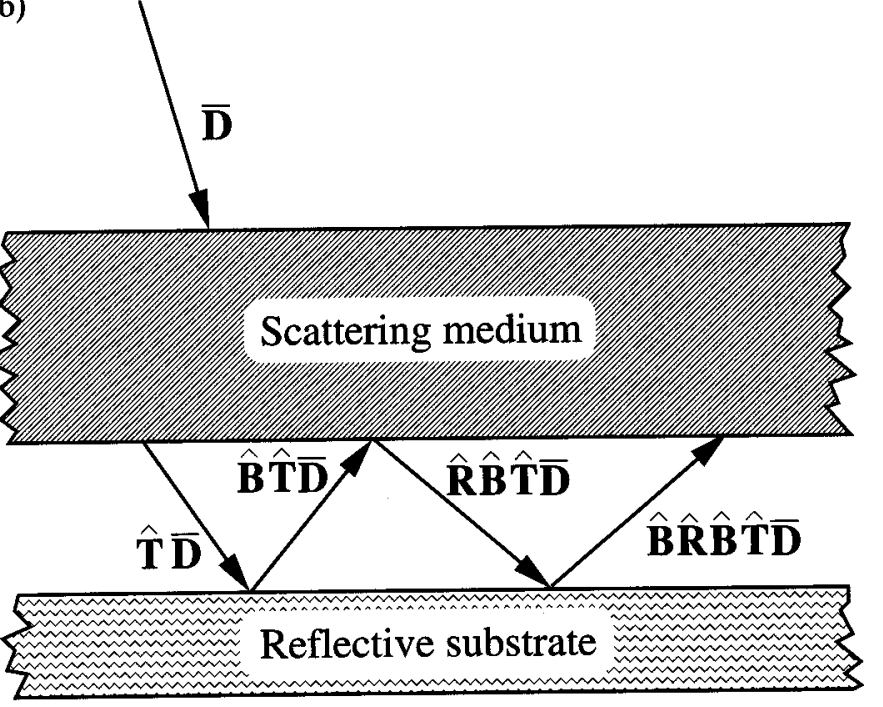

Fig. 1. Schematic illustration of the fuxes or radiances involved in a calculation by the adding method. Part (a) shows the total fluxes that are discussed in the context of Eqns. 3-6. Part (b) shoms some of the components of the total flux $\underline{D}$. 
In reference to Fig. 1(b), the terms in this equation with the various orders of reflection of $\hat{T} \bar{D}$ between the scattering medium and the reflective substrate. The total fluxes $(\underline{D}$ and $\underline{U})$ that are represented in Fig. 1(a) and Eqns. 3-6 are the sums of the components that are shown in Fig. 1(b). Remembering that the various operators are applied from right to left, $\hat{T} \bar{D}$ is the fraction of $\bar{D}$ that penetrates the scattering medium. Of this, a fraction $\hat{B}$ is reflected from the substrate, and of this, a further fraction $\hat{R}$ is reflected back downwards. This yields the term $\hat{R} \hat{B} \hat{T} \bar{D}$ in Eqn. 7. Further reflections add further terms to the total flux $D$, each reflection up then down multiplying the component flux by a further $\hat{R} \hat{B}$.

In the rest of this paper exitances and irradiances (i.e. fluxes) are considered, so the transfer operators become scalar coefficients. However, the above formalism is recommended to those wishing to extend the work. That is, the adding method can be used to calculate the radiative response of the soil-vegetation column even if complicated models are used for the individual components (soil and vegetation).

\section{Nomenclature}

We adopt the following notation and nomenclature. Whether a flux is an irradiance or an exitance is determined by its interaction with the vegetation, as shown in Fig. 2. That is, a flux incident on the vegetation is labelled as an irradiance $(E)$, and a flux exitant from the vegetation is labelled an exitance $(M)$. The symbol $M$ or $E$ is augmented if necessary by a superscripted arrow pointing upwards or downwards, indicating the direction of the flux. Furthermore, fluxes undergo multiple reflections between the vegetation and the soil, as shown in Fig. 2. Fluxes are labelled with a numerical subscript that indicates how many times the radiation has interacted with the vegetation. The subscripted ' $v$ ' indicates that the fluxes in question originated in the vegetation. Subscripts ' $s$ ' and ' $r$ '

\section{Reference}

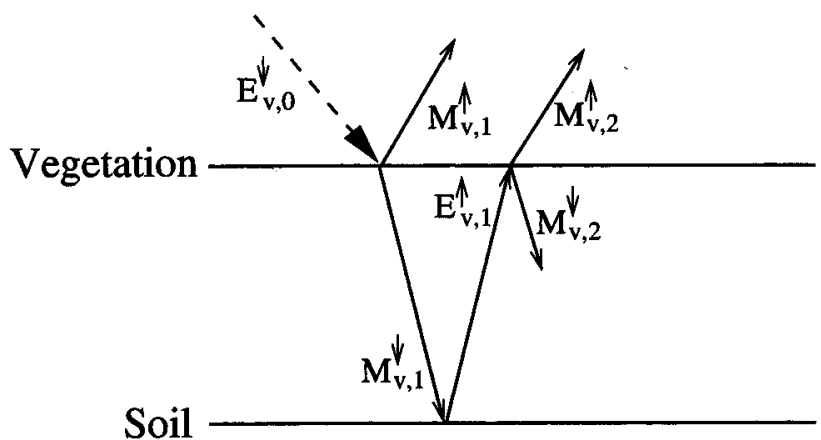

Fig. 2. Schematic illustration of the fate of thermal fuxes emitted by the vegetation. The broken arrow indicates a fictitious flux, invoked to simplify the derivations. indicate fluxes whose origins are in the soil or atmosphere, respectively. Finally, thermal fluxes are written in a plain typeface, shortwave fluxes in bold. This notation is unambiguous.

For scattering and transmission operators, subscripts $s$ and $t$ indicate that the operators are applicable in the shortwave and thermal wavebands, respectively. So $\hat{R}_{t}$ and $\hat{R}_{s}$ are thermal and shortwave reflectances of the vegetation respectively; and similarly with the transmittances $\hat{T}_{t}$ and $\hat{T}_{s}$, and reflectances $\hat{B}_{t}$ and $\hat{B}_{s}$ of the soil.

\section{Scattering coefficients.}

It is convenient to define the following condensation of the notation:

$$
\begin{aligned}
& \epsilon_{s}^{\prime}=1-\epsilon_{s,} \\
& \epsilon_{v}^{\prime}=1-\epsilon_{v,}
\end{aligned}
$$

where $\epsilon$ is the emissivity of the substance specified by its subscript.

Let isotropic thermal radiation of radiance $L_{t}$ illuminate a slab of vegetation of optical depth $\tau$. At an angle $\theta$ from the perpendicular, a radiance $L_{t} e^{-\tau / \mu}$ will be transmitted unintercepted, where $\mu=\cos (\theta)$. The total transmitted, unintercepted exitance is calculated by the formula of equation $\mathrm{A3}$, and is:

$$
2 \pi L_{t} E_{3}(\tau)
$$

where $E_{3}(\cdot)$ is the exponential integral [Abramowitz and Stegun (1972)]. By isotropy and the $\pi$-rule, the irradiance is $\pi L_{t}$, so the intercepted flux is:

$$
\pi L_{t}\left[1-2 E_{3}(\tau)\right]
$$

Of this, a fraction given by the single-scattering albedo $e_{v}^{\prime}$ is scattered equally and isotropically upward and downward. Thus, the reflected flux is:

$$
\frac{1}{2} \epsilon_{v}^{\prime} \pi L_{t}\left[1-2 E_{3}(\tau)\right]
$$

Thus the reflection operator for the flux of isotropic thermal radiation is:

$$
\hat{R}_{t}=\frac{1}{2} \epsilon_{v}^{\prime}\left[1-2 E_{3}(\tau)\right] .
$$

That is, the flux of reflected thermal radiation is given by a fraction $\hat{R}_{t}$ of the incident flux. Similarly, the transmission operator for isotropic thermal radiation is:

$$
\hat{T}_{t}=2 E_{3}(\tau)+\hat{R}_{t}
$$

It is assumed that a fraction $\epsilon_{s}^{\prime}$ of any thermal flux incident on the soil is reflected. Thus, the reflection coefficient for thermal fluxes incident on the soil is:

$$
\hat{B}_{t}=\epsilon_{s}^{\prime} \text {. }
$$

Similarly, the soil is assumed to have a constant albedo $a_{s}$ in the shortwave waveband. Thus the reflection coefficient for shortwave fluxes incident on the soil is: 


$$
\hat{B}_{s}=a_{s} \text {. }
$$

For incident isotropic shortwave radiation, the interception is by assumption the same as for thermal radiation. The single-scattering albedo is now $a_{v}$, and the reflection and scattering operators for shortwave radiation are:

$$
\begin{aligned}
& \hat{R}_{s}=\frac{1}{2} a_{v}\left[1-2 E_{3}(\tau)\right], \\
& \hat{T}_{s}=2 E_{3}(\tau)+\hat{R}_{s} .
\end{aligned}
$$

\section{Derivation of shortwave and thermal fuxes.}

Shortwave and thermal radiative fluxes are considered to originate above the reference height of the SVAT, and thermal fluxes to originate in the soil and vegetation. The adding method derived above is used in each case; but fictitious fluxes must be invoked in some cases to force the problem into the formalism of the adding method. Although this may appear inelegant, it is the easiest way to arrive at the correct results.

i) Thermal fluxes originating in the vegetation

The relevant fluxes are illustrated in Fig. 2. These are component fluxes that are added to form the total fluxes illustrated in Fig. 1. By an assumption of the model, the upward and downward exitances $M_{v, 1}^{\uparrow}$ and $M_{v, 1}^{\downarrow}$ are equalthey are quantified in Eqns. 24 and 28 and in Appendix B. This problem can be solved by invoking an imaginary incident flux, $E_{v, 0}^{\downarrow}$, as shown in Fig. 2. For consistency, we must demand that:

$$
M_{v, 1}^{\downarrow}=\hat{T} E_{v, 0}^{\downarrow}
$$

and thus:

$$
E_{v, 0}^{\downarrow}=\hat{T}_{t}^{-1} M_{v, 1}^{\downarrow}
$$

The transfer operators are merely coefficients, so that inversion is achieved by taking the reciprocal.

Thus, using the adding method (remembering to include $M_{v, 1}^{\uparrow}$ and to omit the fictitious $\hat{R}_{t} E_{v, 0}^{\downarrow}$ ), the total fluxes are:

$$
\begin{aligned}
M_{v, \text { total }}^{\downarrow} & =\left[\hat{I}-\hat{R}_{t} \hat{B}_{t}\right]^{-1} \hat{T} E_{v, 0}^{\downarrow}=\left[\hat{I}-\hat{R}_{t} \hat{B}_{t}\right]^{-1} M_{v, 1}^{\downarrow}, \\
E_{v, \text { total }}^{\uparrow} & =\hat{B}_{t} M_{v, \text { total }}^{\downarrow}, \\
M_{v, \text { total }}^{\uparrow} & =M_{v, 1}^{\uparrow}+\hat{T} E_{v, \text { total }}^{\uparrow} .
\end{aligned}
$$

These and later formulae can be confirmed by expanding the operator $\left[\hat{I}-\hat{R}_{t} \hat{B}_{t}\right]^{-1}$ in the binomial series of Eqn. 7, thus explicitly revealing all orders of reflection between the soil and vegetation.

This analysis must be completed by a specification of $M_{v, 1}^{\uparrow}$ and $M_{v, 1}^{\downarrow}$, the thermal fluxes emitted by the canopy upwards and downwards. The vegetation is a complex three-dimensional structure, not a simple surface, so it is not sufficient to use the Stefan-Boltzmann law $M_{v, 1}^{\uparrow}=$ $\epsilon_{v} \sigma T_{v}^{4}$, where $\sigma$ is the Stefan-Boltzmann constant and $T_{v}$ is the temperature of the vegetation. This can be seen by noting that, although each leaf is assumed to emit thermal radiation of exitance $\epsilon_{v} \sigma T_{v}^{4}$, the whole canopy will emit a smaller quantity related to the density of the vegetation composing it. This effect is modelled by multiplying the exitance by a factor that is a function $f$ of the leaf area index $A_{L}$ :

$$
M_{v, 1}^{\downarrow}=M_{v, 1}^{\uparrow}=f\left(A_{L}\right) \sigma \epsilon_{v} T_{v}^{4}
$$

Clearly,

$$
\begin{aligned}
& \lim _{A_{L} \rightarrow 0} f\left(A_{L}\right)=0, \\
& \lim _{A_{L} \rightarrow \infty} f\left(A_{L}\right)=1,
\end{aligned}
$$

so that a canopy containing no leaves emits no radiation, and one of infinite $A_{L}$ is not perforated. Consideration of the shadowing of leaves by one another leads to a further criterion, that:

$$
\frac{d^{2} f\left(A_{L}\right)}{d A_{L}^{2}}<0 \quad\left(0 \leq A_{L}<\infty\right),
$$

i.e. that $F\left(A_{L}\right)$ increases rapidly with $A_{L}$ when $A_{L}$ is small, but that it approaches its limit of 1 slowly. It is convenient to set

$$
f\left(A_{L}\right)=1-2 E_{3}\left(A_{L} / 2\right),
$$

as this function satisfies Eqns. 25-27, and must be calculated because it is used elsewhere in the model. A further justification of this choice is presented in Appendix B.

ii) Thermal fluxes originating in the soil

The fluxes are labelled as shown in Fig. 3. The analysis is very similar to that above except that the originating flux is $E_{s, 0}^{\uparrow}$. It is assumed that the soil emits isotropic thermal radiation according to the Stefan-Boltzmann law:

$$
E_{s, 0}^{\uparrow}=\sigma \epsilon_{s} T_{s}^{4},
$$

where $T_{s}$ is the temperature of the soil's surface.

A fictitious irradiance, $E_{s, 0}^{\downarrow}$ is required:

$$
E_{s, 0}^{\downarrow}=\hat{T}_{t}^{-1} M_{s, 1}^{\downarrow}=\hat{T}_{t}^{-1} \hat{R} E_{s, 0}^{\uparrow} .
$$

Thus:

$$
\begin{aligned}
M_{s, \text { total }}^{\downarrow} & =\left[\hat{I}-\hat{R}_{t} \hat{B}_{t}\right]^{-1} \hat{R}_{t} E_{s, 0}^{\uparrow} \\
E_{s, \text { total }}^{\uparrow} & =\hat{B}_{t} M_{s, \text { total }}^{\downarrow}+E_{s, 0}^{\uparrow}, \\
M_{s, \text { total }}^{\uparrow} & =\hat{T} E_{s, \text { total }}^{\uparrow} .
\end{aligned}
$$

iii) Thermal fluxes originating in the sky

The fluxes are labelled as in Fig. 4. and the adding method can be applied straightforwardly:

$$
\begin{aligned}
M_{r, \text { total }}^{\downarrow} & =\left[\hat{I}-\hat{R}_{t} \hat{B}_{t}\right]^{-1} \hat{T}_{t} E_{r, 0}^{\downarrow}, \\
E_{r, \text { total }}^{\uparrow} & =\hat{B}_{t} M_{r, \text { total }}^{\downarrow}, \\
M_{r, \text { total }}^{\uparrow} & =\hat{R}_{r} E_{r, 0}^{\downarrow}+\hat{T} E_{r, \text { total }}^{\uparrow} .
\end{aligned}
$$




\section{Reference}

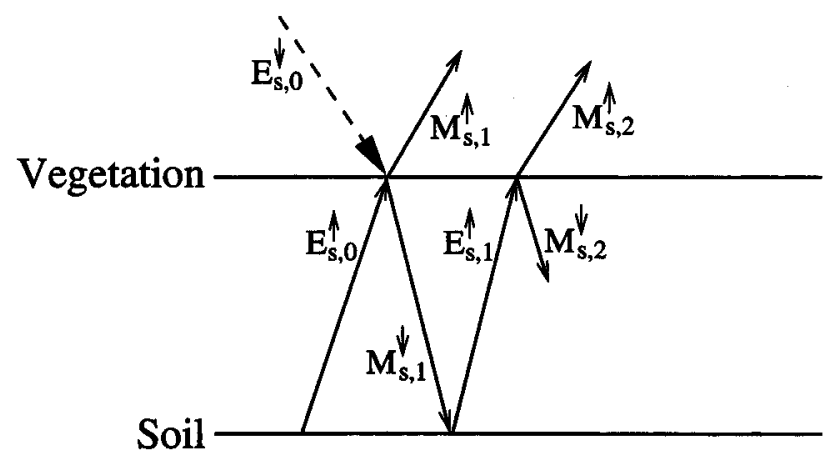

Fig. 3. Schematic illustration of the fate of thermal fluxes emitted by the soil. The broken arrow indicates a fictitious fux, invoked to simplify the derivations.

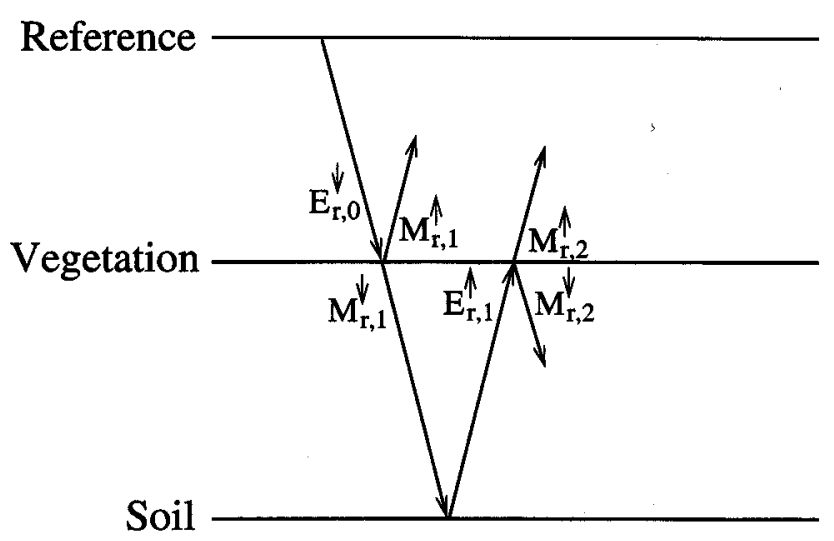

Fig. 4. Schematic illustration of the fate of thermal fuxes incident on the top of the vegetation.

An expression for the emissivity $\epsilon_{a}$ of the atmosphere in the absence of clouds is given by Campbell (1985):

$$
\epsilon_{a}=0.58 e_{a}^{\frac{1}{7}}
$$

where $e_{a}$ is the water vapour concentration in $\mathrm{g} \mathrm{m}^{-3}$ at the screen height. Thus,

$$
E_{r, 0}^{\downarrow}=\epsilon_{a} \sigma T_{a}^{4},
$$

where $T_{a}$ is the temperature at screen height. The validity of formulae such as this has been questioned, but they are accurate enough for most applications of SVATs [Monteith and Unsworth (1990)].

iv) Shortwave fluxes

In Fig. 5, it is assumed that direct-beam solar radiation of radiance $\mathbf{L}_{0}^{\downarrow}($ sun $)$ is incident as if from an infinitely distance point source. If the Sun's zenith-angle is $\theta_{0}=\arccos \mu_{0}$, then the incident flux is given by Eqn. A6:

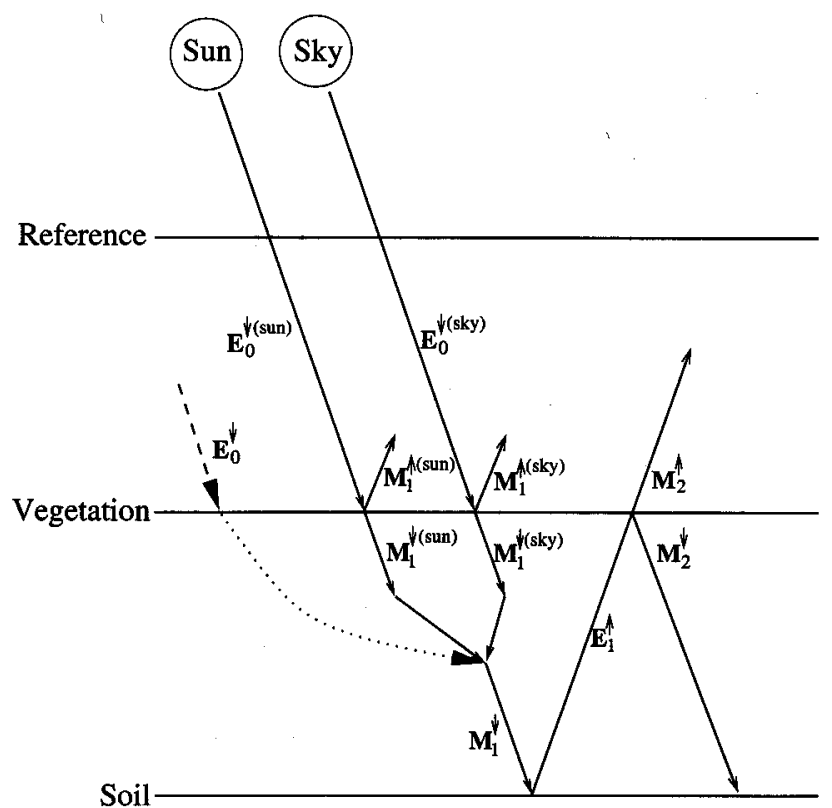

Fig. 5. Schematic illustration of the fate of shortwave fuxes incident on the top of the vegetation. The broken arrow indicates a fictitious flux, invoked to simplify the derivations.

$$
\mathbf{E}_{0}^{\downarrow(s u n)}=\mu_{0} \mathbf{L}_{0}^{\downarrow(s u n)},
$$

and the unintercepted transmitted flux is:

$$
\mathbf{M}_{1}^{\downarrow(\text { sun })}=e^{-\tau / \mu_{0}} \mu_{0} \mathbf{L}_{0}^{\downarrow(s u n)} .
$$

By assumption, a fraction $a_{v}$ of the intercepted flux is scattered isotropically, equally upwards and downwards. Thus:

$$
\begin{aligned}
& \mathbf{M}_{1}^{\uparrow(\text { sun })}=\frac{a_{v}}{2}\left[1-e^{-\tau / \mu_{0}}\right] \mathbf{E}_{0}^{\downarrow_{(\text {sun })}}, \\
& \mathbf{M}_{1}^{\left.\downarrow \downarrow_{\text {sun }}\right)}=\frac{a_{v}}{2}\left[1-e^{-\tau / \mu_{0}}\right] \mathbf{E}_{0}^{\downarrow_{\text {(sun })}}+\mathbf{E}_{0}^{\downarrow(\text { sun })} e^{-\tau / \mu_{0}}
\end{aligned}
$$

Skylight is assumed to be isotropic with an irradiance $\mathbf{E}_{0}^{\downarrow(s k y)}$, and thus:

$$
\begin{aligned}
& \mathbf{M}_{1}^{\downarrow(s k y)}=\hat{T}_{s} \mathbf{E}_{0}^{\downarrow(s k y)}, \\
& \mathbf{M}_{1}^{\uparrow(s k y)}=\hat{R}_{s} \mathbf{E}_{0}^{\downarrow(s k y)} .
\end{aligned}
$$

To cast the problem into a form amenable to the adding method, a fictitious irradiance is invoked:

$$
\mathbf{E}_{0}^{\downarrow}=\hat{T}_{s}^{-1} \mathbf{M}_{1}^{\downarrow}
$$

where

$$
\mathbf{M}_{1}^{\downarrow}=\mathbf{M}_{1}^{\downarrow(s u n)}+\mathbf{M}_{1}^{\downarrow(s k y)}
$$

Thus:

$$
\begin{aligned}
\mathbf{M}_{\text {total }}^{\downarrow} & =\left[\hat{I}-\hat{R}_{s} \hat{B}_{s}\right]^{-1} M_{1}^{\downarrow}, \\
\mathbf{E}_{\text {total }}^{\uparrow} & =\hat{B}_{s} \mathbf{M}_{\text {total }}^{\downarrow}, \\
\mathbf{M}_{\text {total }}^{\uparrow} & =\mathbf{M}_{1}^{\uparrow(s u n)}+\mathbf{M}_{1}^{\uparrow(s k y)}+\hat{T}_{s} \mathbf{E}_{\text {total }}^{\uparrow} .
\end{aligned}
$$


v) Total fluxes

The complete solution is obtained by adding appropriate flux components, as follows.

The total irradiance onto the soil is:

$$
M_{r, \text { total }}^{\downarrow}+M_{s, \text { total }}^{\downarrow}+M_{v, \text { total }}^{\downarrow}+\mathbf{M}_{\text {total }}^{\downarrow} .
$$

The total exitance from the soil is:

$$
E_{r, \text { otal }}^{\uparrow}+E_{s, \text { total }}^{\uparrow}+E_{v, \text { total }}^{\uparrow}+\mathbf{E}_{\text {total }}^{\uparrow} .
$$

The total irradiance onto the vegetation is:

$$
E_{r, \text { total }}^{\uparrow}+E_{s, \text { total }}^{\uparrow}+E_{v, \text { total }}^{\uparrow}+\mathbf{E}_{\text {total }}^{\uparrow}+E_{r, 0}^{\downarrow}+\mathbf{E}_{0}^{\downarrow \downarrow \text { sun })}+\mathbf{E}_{0}^{\downarrow(s k y)} .
$$

The total exitance from the vegetation is:

$$
\begin{array}{r}
M_{r, \text { total }}^{\uparrow}+M_{s, \text { total }}^{\uparrow}+M_{v, \text { total }}^{\uparrow}+\mathbf{M}_{\text {total }}^{\uparrow}+M_{r, \text { total }}^{\downarrow}+\mathbf{M}_{s, \text { total }}^{\downarrow} \\
M_{v, \text { total }}^{\downarrow}+\mathbf{M}_{\text {total }}^{\downarrow} .
\end{array}
$$

The total reflected shortwave flux is:

$$
\mathbf{M}_{\text {total }}^{\uparrow} \text {. }
$$

The net radiation is:

$$
\begin{aligned}
E_{r, 0}^{\downarrow}+\mathbf{E}_{0}^{\downarrow \text { (sun })}+ & \mathbf{E}_{0}^{\downarrow(s k y)}- \\
& {\left[M_{r, \text { total }}^{\uparrow}+M_{s, \text { total }}^{\uparrow}+M_{v, \text { total }}^{\uparrow}+\mathbf{M}_{\text {total }}^{\uparrow}\right] . }
\end{aligned}
$$

Although this array of equations appears complex, each is formed merely by the addition of the basic fluxes derived earlier in the paper. In turn, these contain only functions that are found in standard mathematical FORTRAN or C libraries, with the exception of the exponential integral $E_{3}(\cdot)$. But there is a very fast and accurate algorithm for computing it [Press et al. (1992)]; it need be computed only once and the result stored in a variable for repeated use in the various formulae that appear in the model. Thus, the model can be executed very efficiently on a computer.

\section{Application}

SWEAT has been discussed in the literature [e.g. Burke (1997), Burke et al. (1998), Daamen (1993, 1997)], and does not require further validation here. However, as RM calculates thermal radiative fluxes that are determined by the temperatures of the vegetation and soil, it cannot be run without the use of a parallel program to estimate these. Indeed, that program and RM must be mutually consistent. For this reason, RM has been woven into SWEAT to form a combined model SWEAT 2.

\section{INTERNAL CONSISTENCY}

Initially, purely artificial runs of SWEAT 2 were performed, to ensure that it generates results qualitatively consistent with experience for a completely specified system. Simulated time series of hourly meteorological data were generated for an 80-day period, to provide upper boundary conditions for SWEAT. Relative humidity and air temperature were assumed to be sinusoidal with a 24 hour period, and a phase lag was assumed to produce a maximum air temperature shortly after noon. Air temperature swung between $22.5^{\circ} \mathrm{C}$ and $32.5^{\circ} \mathrm{C}$, relative humidity between $45 \%$ and $95 \%$. Wind speed in $\mathrm{m} \mathrm{s}^{-1}$ was drawn from a uniform random distribution on $[0,1]$, cubed to skew it towards lower values, and multiplied by 5 to give a maximum of $5 \mathrm{~m} \mathrm{~s}^{-1}$. At each hourly time step, a uniform random deviate on $[0,1]$ was drawn, and if it was above a certain threshold, it was assumed that a number of millimetres of rain fell, drawn from another uniform random deviate on $[0,5]$. On the following time-step, the probability of rain was increased if rain had fallen on the previous time-step, thus increasing the average length of rainy periods. Finally, insolation was calculated from a simple solar declination model, assuming a latitude of $28.6^{\circ}$ North, and solar noon of 12.00 local time. A simple atmospheric attenuation was modelled as the negative exponential of the optical depth divided by the solar zenith angle, with an optical depth arbitrarily sat as 0.3 . The shortwave irradiance at the top of the atmosphere was assumed to be $1360 \mathrm{~W} \mathrm{~m}^{-2}$. The incident shortwave radiation was partitioned between the direct solar beam and isotropic skylight according to a simple exponential attenuation law, where the flux lost by attenuation from the direct solar beam was assigned to the isotropic skylight. Short samples of simulated rainfall and solar irradiance are illustrated in Fig. 6.

The vegetation was assumed to have a leaf area index $A_{L}$ $=0.5$ in the first simulation and, $A_{L}=5.0$ in the second. These will be referred to as the SWEAT $2 / 0.5$ run and the SWEAT 2/5.0 run, respectively. The soil profile was initialized at a uniform temperature of $25.0^{\circ} \mathrm{C}$ and the volumetric water content of $16.1 \%$ was uniform from the surface down to a depth of $3.6 \mathrm{~m}$. The soil had an albedo $a_{s}=0.3$, the vegetation a single-scattering albedo $a_{v}=0.18$. The vegetation had an emissivity $\epsilon_{v}=0.97$ and the soil $\epsilon_{s}=0.95$.

The 80-day runs of SWEAT 2 produced simulated time series of net radiation as outputs. Part of the time series of $R_{n}$ from the SWEAT 2/0.5 run is shown in Fig. 6. These simulated net radiation time series were then used with the same meteorological data and soil and canopy parameters as the SWEAT 2/0.5 and SWEAT 2/5.0 runs, in calculations referred to as the SWEAT 1/0.5 and SWEAT 1/5.0 runs. For these, SWEAT was used with its original Beer's Law partitioning of $R_{n}$, as in Eqns. 1 and 2 .

In all runs, the rainfall was not enough to maintain the water content of the profile, which dried out through the 80 day runs.

The behaviour of the partitioning of net radiation between the SWEAT 1 and SWEAT 2 runs was qualitatively different. This is shown in Fig. 7(a) for the runs at $A_{L}=0.5$, and Fig. 7(b) for the runs at $A_{L}=5.0$. In each graph, the horizontal lines indicate the fraction of $R_{n}$ avail- 


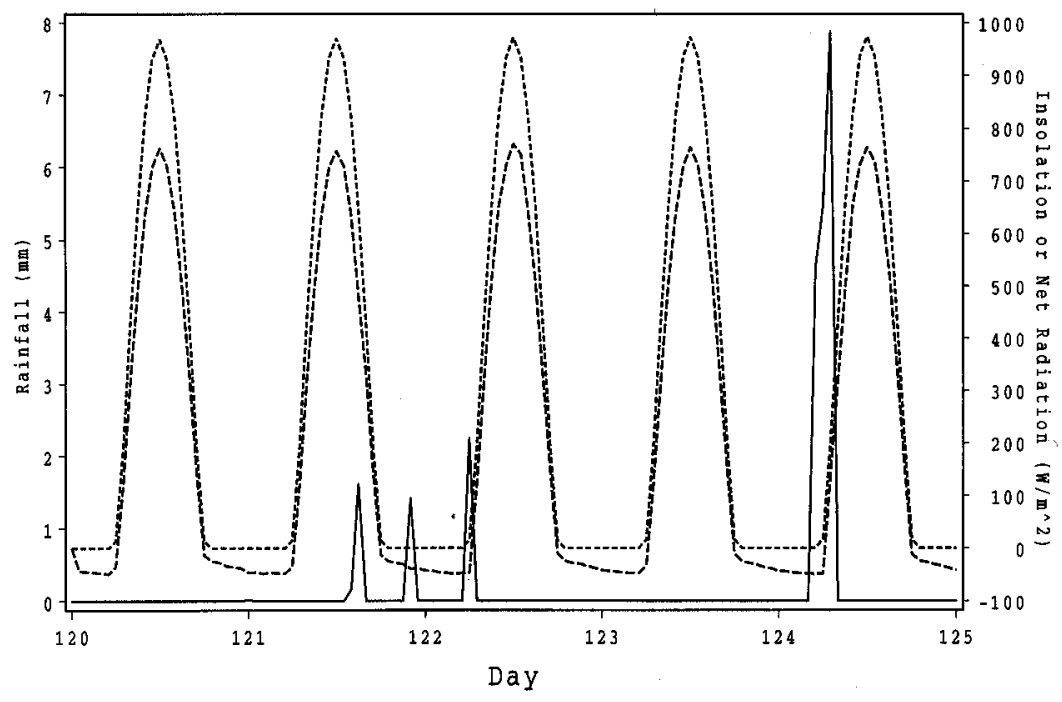

Fig. 6. Part of simulated time series of rainfall, solar irradiance and net radiation. Net radiation was simulated by the $S W E A T$ 2 model, using the solar fux shown here as input. This time series of net radiation was then used to drive the old formulation of SWEAT, in the SWEAT 1/0.5 and SWEAT 1/5.0 runs.

able to the soil as calculated by SWEAT using the formulation of Eqns. 1 and 2. By definition, this does not vary with time. Also in each graph, the vertical bars show one standard deviation of the fraction of $R_{n}$ that is available to the soil, at a given hour over each of the 80 days of the SWEAT 2 runs. As expected, the fraction of $R_{n}$ that is available to the soil is highest at noon, when the sun is closest to the zenith. In the runs at $A_{L}=0.5$, although the total net radiation is the same for the SWEAT 2 and SWEAT runs, the fraction reaching the soil is systematically lower as calculated by SWEAT 2 , as well as having diurnal variation. In these cases, the leaf area index is low, so the radiation transport is dominated by the soil. That is, a relatively large amount of shortwave radiation is absorbed by the soil, and the high surface temperature reduces $R_{n}$ by thermal radiation. By contrast, in the runs with high leaf area index, the radiation is dominated by the vegetation. The vegetation insulates the soil against thermal emission into the atmosphere, with a consequent increase in the fraction of $R_{n}$ that is available to the soil, as shown in Fig. 7(b).

The differences between heat fluxes into the atmosphere between the two runs at low- $A_{L}$ are significant, but not great; in both runs, the instantaneous available energy flux $\left(R_{n}\right)$ is the same at all times. Figure 8 shows this for these low- $A_{L}$ runs. The two runs diverge at the start when the profile is wet, but converge again as it dries out. It is not possible to apply intuitive reasoning here, as the partitioning of radiation between vegetation and soil has complex effects on evaporation from the soil surface, and on transpiration as mediated by stomatal resistance. However, the evaporative fraction reduces as the profile dries out, as one would expect.

Figure 8(c) shows that the soil heat flux is higher for the SWEAT 1/0.5 than for the SWEAT $2 / 0.5$ run, because the fraction of $R_{n}$ that is available to the soil is greater in the former. This is confirmed in Fig.8(d), which shows that soil surface temperature at midnight and noon is greater in the SWEAT 1/0.5 run than in the SWEAT $2 / 0.5$ run.

As discussed above, when $A_{L}=5.0$, SWEAT 2 gave a greater fraction of $R_{n}$ to the soil than did SWEAT. This is confirmed in Fig. 9(a), which shows that SWEAT 2 led to a higher soil heat flux. Further confirmation is provided by Fig. 9(b), showing that SWEAT 2 resulted in higher soil surface temperatures.

\section{SIMULATIONS}

SWEAT is designed to predict fluxes accurately for a sparse canopy, so the radiation component of SWEAT 2 has been tested against data gathered over a millet crop at the Southern Supersite of the HAPEX-Sahel experiment [Goutorbe et al. (1997); Wallace et al. (1993)]. These data consist of time series of the radiative and meteorological conditions; profiles of root-length density; relevant fluxes; and leaf area index. All of these data are to be found in the HAPEX-Sahel Information System on the World Wide Web at

http://www.orstom.fr/hapex 
(a)

$\mathrm{LAl}=0.5$

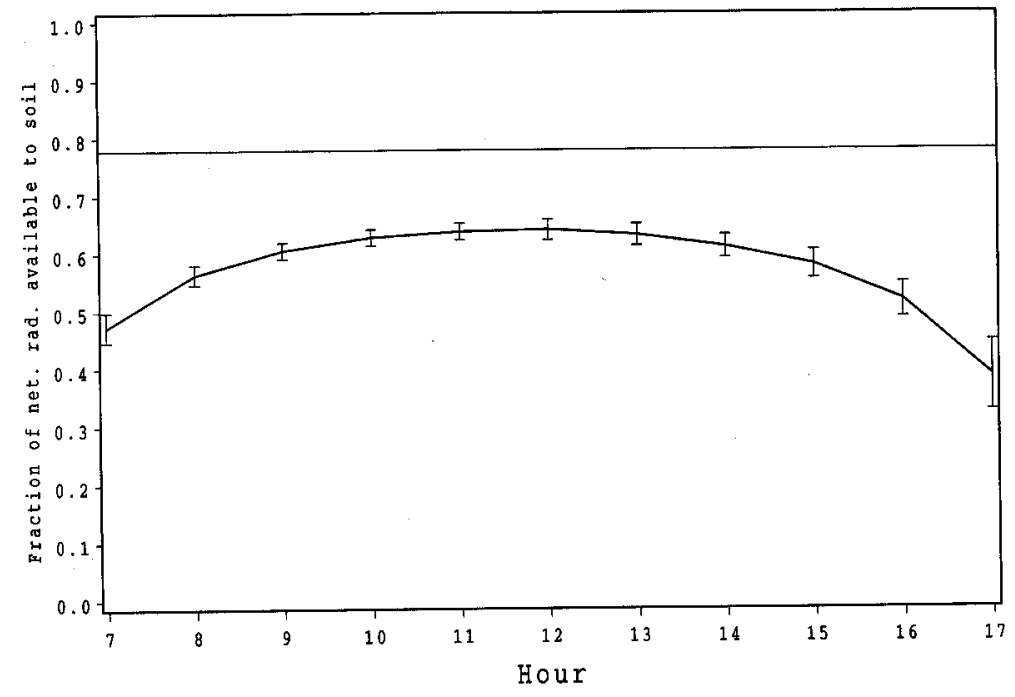

(b)

$$
L A I=5.0
$$

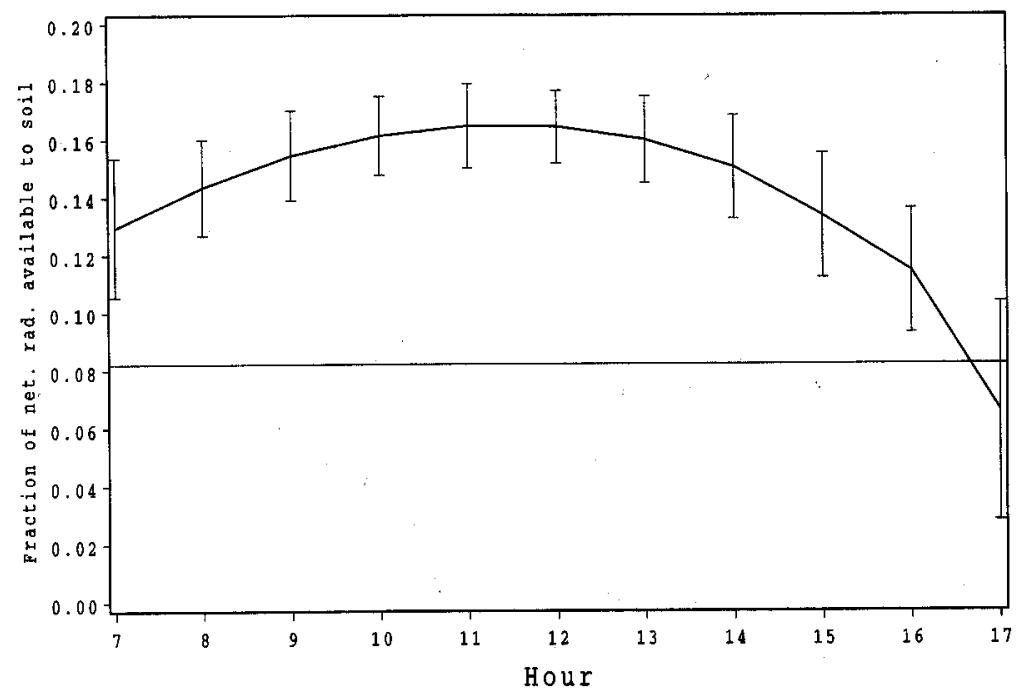

Fig. 7. In part (a), the horizontal reference line indicates $R_{s} / R_{n}$, the fraction of net radiation that is available to the soil, using Beer's law when $A_{L}=0.5$. The vertical bars show the value of this quantity as simulated by $R M$ in $S W E A T 2$, over an 80-day period. Part (b) is as part (a), except $A_{L}=5.0$.

except the data on roots which were supplied by one of the authors (L.P.S).

Initial profiles of soil water potential and temperature were estimated by running the model from a long, arbitrary time before the period of interest, starting from arbitrary but plausible profiles.

The thermal emissivity of the soil was assumed to be constant at 0.90 , that of the vegetation was set at 0.97
[Monteith and Unsworth (1990)]. The radiative interception efficiency of millet has been determined experimentally by Wallace et al. (1986) to be 0.41 . Thus, and in accordance with the discussion at the end of Appendix B, the optical depth $\tau$ in Eqn. B2 was replaced by

$$
\tau=0.41 A_{L} .
$$

The shortwave optical properties of the soil and vegeta- 
(a)

$$
L A \mid=0.5
$$

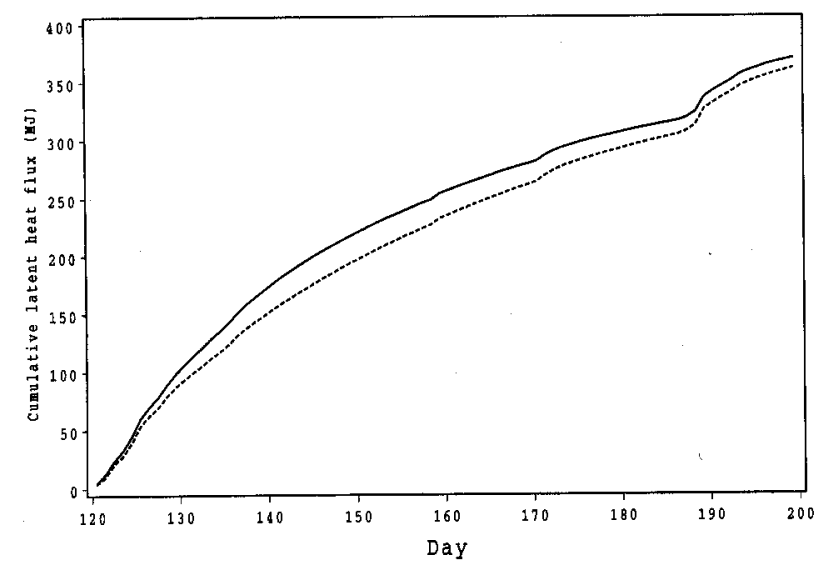

plom

(c)

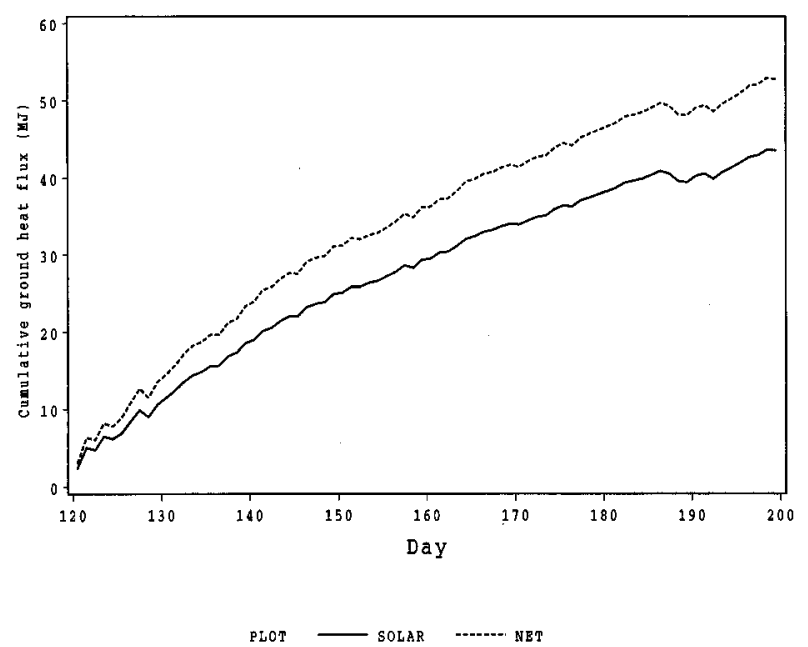

(b)

$L A \mid=0.5$

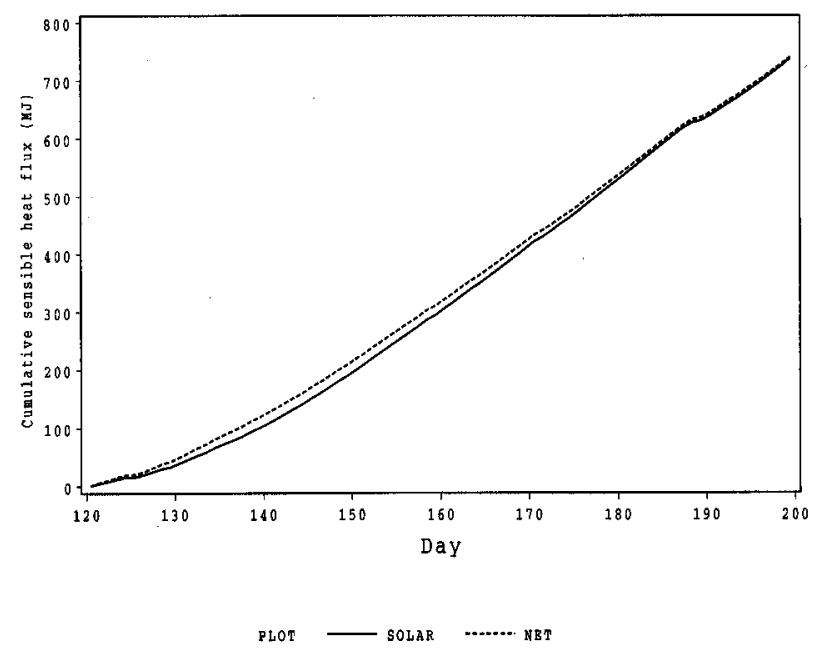

(d)

$L A l=0.5$

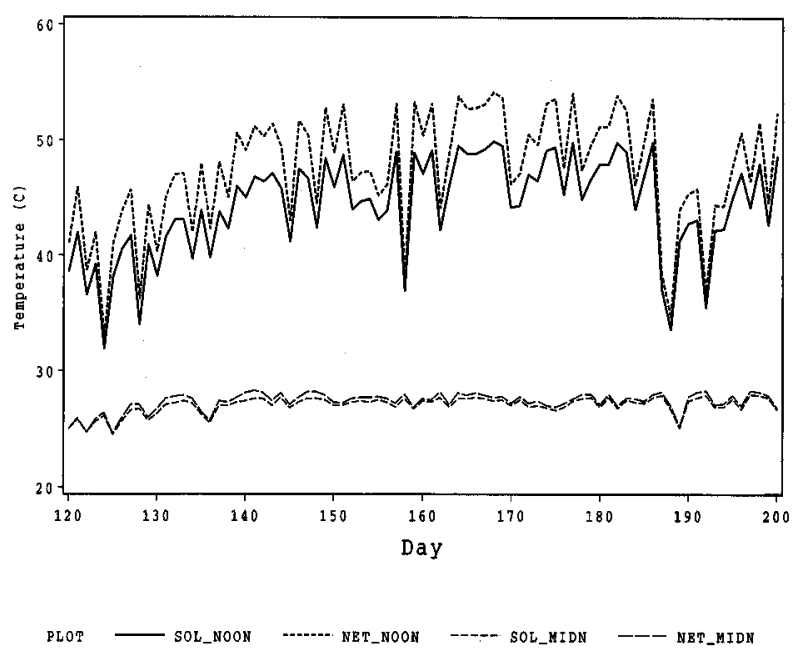

Fig. 8. Results of 80 day runs of SWEAT 2, using artificial meteorological data with $A_{L}=0.5$. In one run, shortwave irradiance was the driving energy source. This run simulated net radiation, which was used in the second. In parts (a-c) of this figure, the solid curve represents the SWEAT 2/0.5 run, the broken curve represents the SWEAT 1/0.5 run. Part (a) shows timeintegrated simulated latent heat fux into the atmosphere. Part (b) similarly shows cumulative sensible heat fux, part (c) cumulative ground heat flux. Observe that the ordinate in part (c) has a much smaller span than in parts (a) and (b). In parts (ac), small diurnal wiggles have been smoothed out by sampling only at noon each day. Part (d) shoms soil surface temperatures at noon and midnight for both runs. The curves, starting with the top one and moving down the graph represent respectively: SWEAT 1/0.5 at noon; SWEAT 2/0.5 at noon; SWEAT 1/0.5 at midnight; SWEAT 2/0.5 at midnight.

tion were allowed to float in an optimisation process to generate the best results over the period DOY $247-249$ in 1992. That is, the optical properties as estimated in the field were not used. The optimal parameters were found to be $a_{s}=0.37, a_{v}=0.34$, and $A_{L}=0.48$. This compares with a measured green leaf area index of 0.36 at DOY 247 in 1992, and a complete LAI of 0.661; and green LAI of 0.46 on DOY232 (complete LAI on DOY 232 is not available). In view of the great spatial variability of $A_{L}$, the modelled value of 0.48 may be considered as consistent with the true value.

The observed and optimal simulated time series of 
(a)

$\lfloor\mathrm{A} I=\mathbf{5 . 0}$

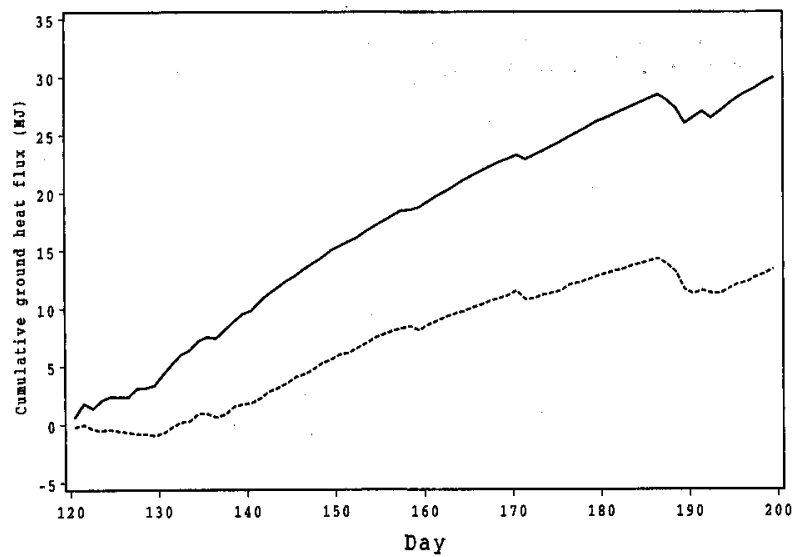

PLOT LOLAR W...... HET (b)

$L A I=5.0$

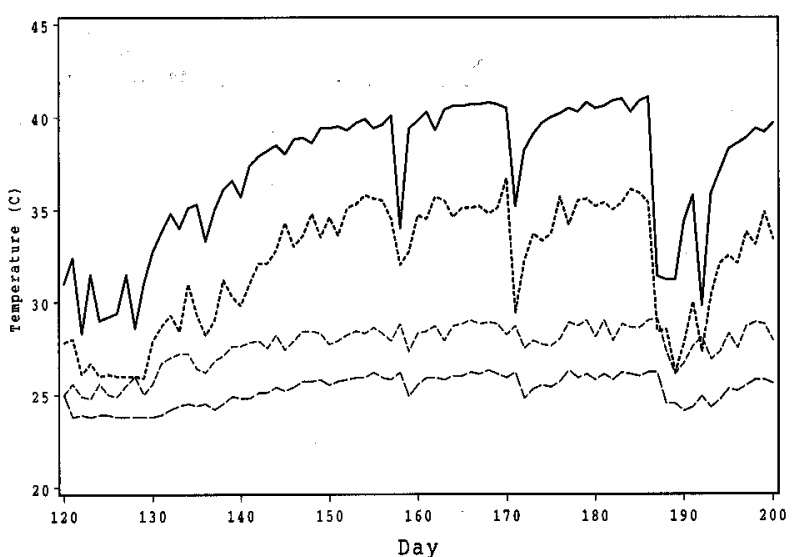

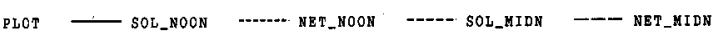

Fig. 9. Parts (a) and (b) correspond to Figs. $8(c)$ and $8(d)$, respectively, except that in this case $A_{L}=5.0$. In part (a) of this figure, the solid curve represents the SWEAT 2/5.0 run, the broken curve represents the SWEAT 1/5.0 run. For part (b), the curves, starting with the top one and moving down the graph represent respectively: SWEAT 2/5.0 at noon; SWEAT 1/5.0 at noon; SWEAT 2/5.0 at midnight; SWEAT 1/5.0 at midnight.

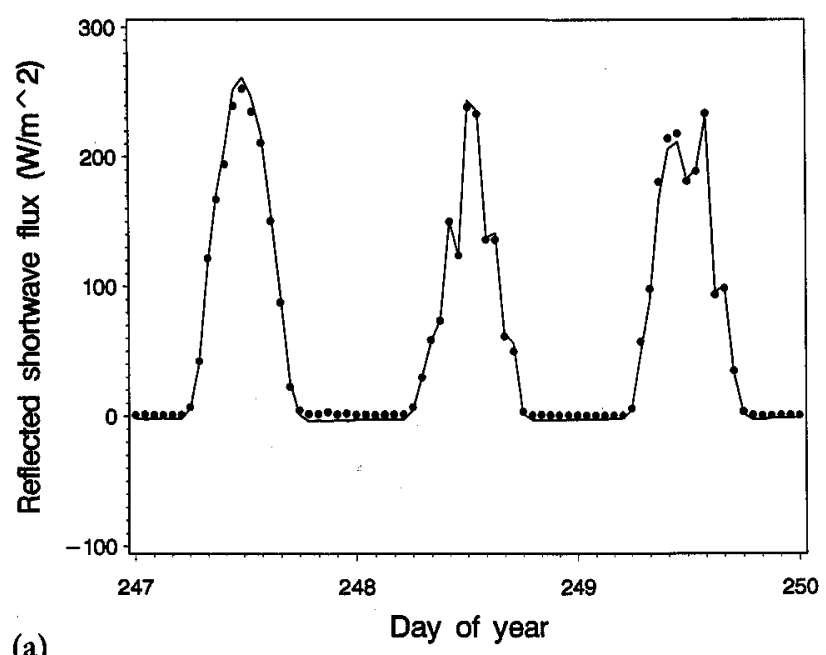

Fig. 10. Part (a) shoms the optimal simulation of the shortwave flux reflected from the millet canopy, as simulated by SWEAT 2 mith $A_{L}=0.48, a_{s}=0.37$ and $a_{v}=0.34$ (curve) and observed experimentally (points). Error bars are too small to be shown on the points. Part (b) shows net radiation above the canopy from the same simulation (curve) and observed experimentally (points). Error bars are too small to be shown on the points. Part (c) shows total heat fux above the canopy from the same simulation (curve) and observed experimentally (points). An apparent outlier in the experimental data in the middle of DOY 247 has been omitted.

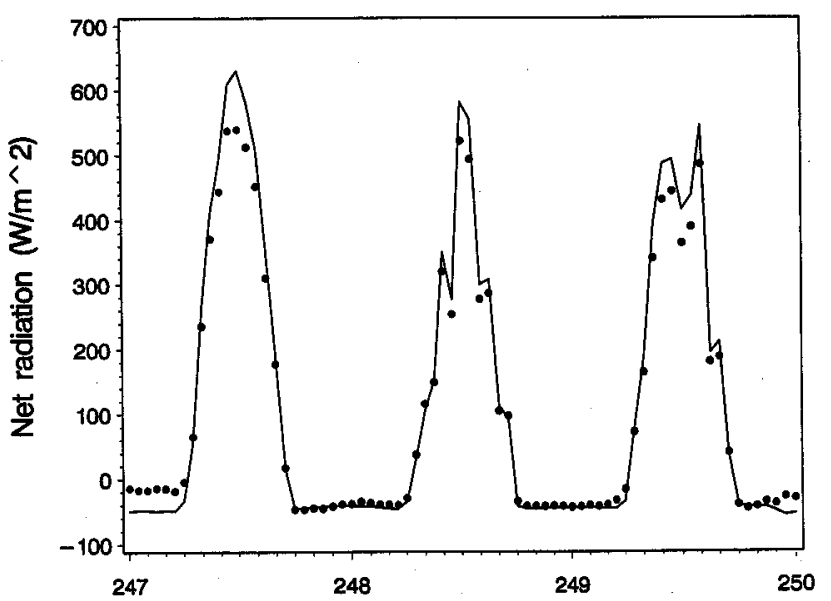

(b)

Day of year

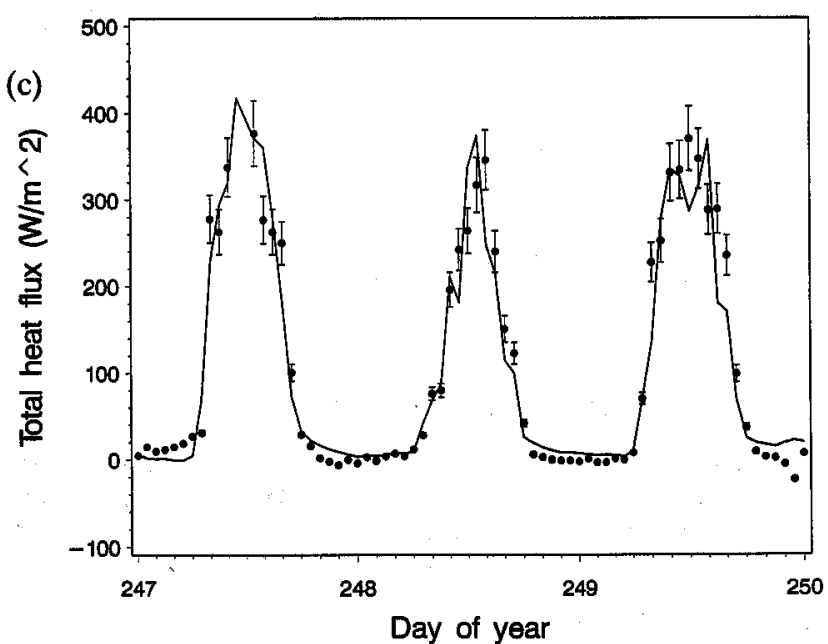


$\mathbf{M}_{\text {total }}^{\uparrow}$ (i.e. reflected shortwave radiation flux above the vegetation) are shown in Fig. 10(a). Figure 10(b) similarly shows the observed and optimal simulated time series of $R_{n}$ above the millet crop. The simulation systematically underestimates $R_{n}$ by about $10 \%$ at mid-day. The total heat flux into the atmosphere is reproduced well, as shown in Fig. 10(c). As the shortwave energy balance is simulated very accurately, the shortfall in $R_{n}$ must be compensated by too small a soil heat flux during the middle of the day. This is consistent with the fact that radiative responses to the horizontal heterogeneity of the canopy must also be greatest in the middle of the day, when the Sun is closest to the zenith.

\section{Discussion and conclusions}

A model (RM) for the interaction of shortwave and thermal radiation with soil and vegetation has been created, for use within a SVAT. The model is conceptually and mathematically simple (although the derivations are lengthy), so it is not computationally expensive. Beer's law requires measurement of net radiation and determination of two parameters: $A_{L}$ and an interception efficiency $\tau / A_{L}$. The new model requires measurement of downwelling shortwave radiation and its partitioning between direct beam and sky light; and determination of four additional parameters: $\epsilon_{v}$, $\epsilon_{s}, a_{v}$, and $a_{s}$. In practice, rough estimates of $\epsilon_{v}, \epsilon_{s}$ are adequate, as is a very crude assumption about the directional partitioning of incident sunlight. The model predicts net radiation and reflected shortwave flux and, when operated as part of a SVAT, enables heat flux into the atmosphere to be estimated well. The only significant problem with RM is its underestimation of solar heating of the soil's surface under a clumped canopy around mid-day.

Although the radiative and heat fluxes are simulated well, not all of the parameters in the optimised model are physically realistic. In this case of $A_{L}=0.48$, good simulations of $\mathbf{M}_{\text {total }}^{\uparrow}$ and $R_{n}$ could be obtained only by setting $a_{s}=0.37$ and $a_{v}=0.34$. As noted by Wallace et al. (1990), 0.37 is a reasonable value for $a_{s}$; but 0.34 is an unrealistically high value for $a_{v}$. At a more realistic lower value, the simulated canopy would absorb more shortwave radiation, overly increasing $R_{n}$ and decreasing $\mathbf{M}_{\text {total }}^{\uparrow}$. In simulations, this could be compensated by decreasing $A_{L}$ to an unrealistic value, which made it impossible to simulate the atmospheric heat flux adequately. This is probably a result of attempting to simulate the radiative properties of a very clumpy canopy by a horizontally homogeneous model. This is consistent with physical intuition; the lower parts of the clumped vegetation contribute little to radiative effects, particularly during the middle of the day when they are maximally shaded by the upper parts. Thus, a given leaf area index in a clumped canopy will have similar radiative effects to a lower LAI in a horizontally homogeneous canopy, just as was found in the use of RM. Despite this difficulty, the new model has advantages of simplicity of inputs and physical realism under many circumstances, and presents an attractive alternative to the formulations usually used in SVATs. The systematic difference between the modelled partitioning of net radiation and that modelled by Beer's law is significant; the diurnal variation of the partitioning in the new model more so. Experiments will now be performed to provide the consistent datasets that will allow a more complete test of the coupled SVAT and radiation model.

\section{Appendix A: radiometric nomenclature, notation and conventions}

The conventions and notation of Nicodemus et al. (1977) are followed. In the rest of the paper, the notation is augmented by arrows and subscripts to distinguish between upwelling and downwelling radiation, and between different orders of interaction with the canopy.

The total radiative flux exiting from a surface is known as the exitance $M$, and the total radiative flux incident on a surface is the irradiance $E$. Both have units of $\mathrm{W} \mathrm{m}^{-2}$ in the S.I. system. A more general radiometric quantity is the radiance, $L$, with units $\mathrm{W} \mathrm{m}^{-2} \mathrm{sr}^{-1}$. It is defined such that, if a spherical polar system of co-ordinates is set up with its equatorial plane coincident with a flat surface, then the irradiance and exitance are given by:

$$
\begin{aligned}
E & =\int_{0}^{2 \pi} \int_{0}^{\pi / 2} L_{i n}(\theta, \phi) \cos \theta \sin \theta d \theta d \phi \\
& =\int_{0}^{2 \pi} \int_{0}^{1} L_{i n}(\mu, \phi) \mu d \mu d \phi,
\end{aligned}
$$

where $L_{\text {in }}(\theta, \phi)$ is the incident radiance, and the substitution has been made

$$
\mu=\cos \theta
$$

and

$$
\begin{aligned}
M & =\int_{0}^{2 \pi} \int_{0}^{\pi / 2} L_{e x}(\theta, \phi) \cos \theta \sin \theta d \theta d \phi \\
& =\int_{0}^{2 \pi} \int_{0}^{1} L_{e x}(\mu, \phi) \mu d \mu d \phi,
\end{aligned}
$$

where $L_{e x}(\mu, \phi)$ is the radiance exitant from the surface.

If the incident or exitant radiation is isotropic, i.e. $L_{e x}(\theta, \phi)$ or $L_{i n}(\theta, \phi)$ is a constant $L_{0}$, then the ' $\pi$-rule' is obtained by evaluation of the integrals in Eqns. $A 1$ and $A 3$ :

$$
\begin{aligned}
M & =\pi L_{0}, \\
E & =\pi L_{0} .
\end{aligned}
$$

If the incident radiance is a Dirac delta-function, i.e. $L_{i n}(\theta, \phi)=L_{0} \delta\left(\phi-\phi_{0}\right) \delta\left(\mu-\mu_{0}\right)$, then the irradiance is

$$
E=L_{0} \cos \theta_{0}=L_{0} \mu_{0} .
$$

A Lambertian surface is defined as one that isotropically reflects all radiation falling onto it. That is, whatever the 
angular distribution of the incident radiation, the reflected radiation is isotropic. In this paper, this definition is generalised (as is commonly done) to say that a Lambertian surface isotropically reflects a fraction $a_{s}$ of the incident flux. That is, $a_{s}$ is the ratio of the exitance to the irradiance, called shortwave albedo. Note that this is a constant (by assumption), whereas real surfaces have an albedo which varies with the angular distribution of incident radiation, even when the surface is physically unaltered [Nicodemus et al. (1977)].

\section{Appendix B: Thermal emission from a homogeneous, isotropic canopy}

That $f\left(A_{L}\right)=1-2 E_{3}\left(A_{L} / 2\right)$ has a prima facie plausibility is evident. Here, a more rigorous argument is presented in its favour. Let us approximate the physics of this problem by substituting for the canopy a homogeneous, isotropically-scattering medium, emitting radiation homogeneously and isotropically with some sourcestrength $S$. Such a problem is amenable to the methods of radiative transfer [Chandrasekhar (1960); Busbridge (1960)]. According to Busbridge, the radiance $L$ in such a medium obeys the following integrodifferential equation:

$$
\mu \frac{\partial L(\mu, t)}{\partial t}=L(\mu, t)-\frac{a_{v}}{2} \int_{-1}^{1} L\left(\mu^{\prime}, t\right) d \mu^{\prime}-S,
$$

where $t$ is the incremental optical depth of the medium, which has a total optical depth of $\tau$.

If, now, the canopy is composed of similar 2dimensional leaves, oriented randomly according to a spherical distribution function (i.e. the probability density function describing the directions of leaf-normal vectors is isotropic), then, for a layer of a given thickness, the layer's optical depth and leaf-area index are related as follows:

$$
\tau=\frac{A_{L}}{2}
$$

[Monteith and Unsworth (1990)]. This implies that a ray of light incident on the canopy will be intercepted with a probability equal to the factor by which a beam would be attenuated if it were incident on a homogeneous medium of optical depth given by Eqn. B2. Further, if the thermal exitance from a leaf is $M_{0}$, it can be shown that the sourcestrength is given by

$$
S=\frac{M_{0}}{\pi}
$$

Busbridge gives a solution to Eqn. B1 in her $\S 42.2$ and $\$ 42.1$. She shows that the radiance exitant from the top and bottom of the canopy is given by:

$$
L(0, \mu)=L(\tau, \mu)=\frac{X(\mid \mu)-Y(|\mu|)}{1-a_{v}} S\left(1-x_{0}-y_{0}\right)
$$

where $X(\mu)$ and $Y(\mu)$ are special functions (defined by Chandrasekhar and Busbridge) which depend on $a_{v}$ and $\tau$; $x_{n}$ and $y_{n}$ are constants given by:

$$
x_{n}=\frac{a_{v}}{2} \int_{0}^{1} X(\mu) \mu^{n} d \mu, \quad y_{n}=\frac{a_{v}}{2} \int_{0}^{1} Y(\mu) \mu^{n} d \mu .
$$

Thus, the exitances from the bottom and top of the canopy can be calculated using Eqns. A1 and A3, and are given by:

$$
\hat{M}=\frac{4 \pi S}{a_{v}\left(1-a_{v}\right)}\left(1-x_{0}-y_{0}\right)\left(x_{1}-y_{1}\right),
$$

where $\hat{M}$ denotes the exact value of the exitance.

Our numerical experiments indicate that $M_{0}\left[1-2 E_{3}(\tau)\right]$ is a good approximation to $\hat{M}$ when $a_{v}<0.1$, but diverges significantly as $a_{v}$ increases, for many plausible values of $\tau$. To understand this, let $a_{v}=0$ in Eqn. B1. Thus:

$$
\mu \frac{\partial L(\mu, t)}{\partial t}=L(\mu, t)-S .
$$

This has solutions of the form

$$
L(\mu, t)=\text { constant } \times e^{t / \mu}+S .
$$

Boundary conditions are obtained by noting that no radiation is incident on the top or bottom of the canopy-we are only considering the effects of the thermal source within the canopy. Thus:

$$
\begin{aligned}
& L(0, \mu)=0, \quad(\mu \leq 0) \\
& L(\tau, \mu)=0, \quad(\mu>0) \text {. }
\end{aligned}
$$

Hence:

$$
\begin{array}{ll}
L(t, \mu)=S\left[1-e^{-\tau / \mu}\right], & (\mu \leq 0) \\
L(t, \mu)=S\left[1-e^{-\left(\tau_{1}-\tau\right) / \mu}\right], & (\mu>0) .
\end{array}
$$

Thus, the exitances at the top and bottom of the canopy are given by:

$$
\begin{aligned}
\hat{M} & =2 \pi S\left[\frac{1}{2}-E_{3}(\tau)\right] \\
& =M_{0}\left[1-2 E_{3}(\tau)\right] .
\end{aligned}
$$

Now the emissivity of vegetation is typically in the range $0.95<\epsilon_{v}<0.99$, [Monteith and Unsworth (1990)] so the single-scattering albedo $\epsilon_{v}^{\prime}$ is small, and it is thus reasonable to use Eqn. 28. This result is equivalent to treating the vegetation as a thin, perforated layer, with a specific area given by $f\left(A_{L}\right)$.

One may wish to replace equation $\mathrm{B} 2$ by a more accurate result based on the true leaf-angle distribution function of the vegetation; or, indeed, by some other ad hoc formula. The same is true of the scattering and interception terms in the transfer operators.

\section{Acknowledgements}

This work was supported largely by Grant No. GST/02/603 under the TIGER programme of the UK Natural Environment 
Research Council. Discussions with Dr. E.J. Burke of the Institute of Hydrology (UK), Dr. S. Gaze of the University of Cambridge Experimental Farm (UK), and Dr. C. Godsalve of the Environmental Systems Science Centre (University of Reading, UK) contributed to the development of this paper. Flux and meteorological data acquired by Dr. C.R. Lloyd and Dr. J.H.C. Gash of the Institute of Hydrology; and LAI data acquired by Dr. P. Levy of the University of Edinburgh (UK) are gratefully acknowledged. The authors would like to thank three anonymous referees for their constructive criticism.

\section{References}

Abramowitz, M.A. and Stegun, I.A., 1972. Handbook of Mathematical Functions with Formulas, Graphs and Mathematical Tables. Dover Publications Inc., London, 9th Printing. SBN 486612724.

Braud, I., Dantas-Antonino, A.C., Vauclin, M., Thony, J.L. and Ruelle, P., 1995. A Simple Soil-Plant-Atmosphere Transfer Model (SiSPAT) Development and Field Verification. 7 . Hydrol. 166, 213-250.

Burke, E.J., 1997. Using a Modelling Approach to Predict Soil Hydraulic Parameters from Passive Micromave Measurements for both Bare and Cropped Soils. Thesis R7397, University of Reading, Reading, England.

Burke, E.J., Gurney, R.J., Simmonds, L.P. and O'Neill, P.E., 1998. Using a Modelling Approach to Predict Soil Hydraulic Properties from Passive Microwave Measurements. IEEE Trans. Geosci. Remote Sensing 36, 454-462.

Busbridge, I.W. 1960. The Mathematics of Radiative Transfer. Cambridge University Press.

Campbell, G.S. 1985. Soil Physics with Basic-Transport Models for Soil-Plant Systems. Elsevier, Amsterdam.

Chandrasekhar, S., 1960. Radiative Transfer. Dover Publications Inc., New York.

Choudhury, B.J., Idso, S.B. and Reginato, R.J., 1987. Analysis of an Empirical Model for Soil Heat Flux Under a Growing Wheat Crop for Estimating Evaporation by an Infrared Temperature Based Energy Balance Equation. Agric. For. Meteorol. 39, 283-297.

Choudhury, B.J. and Monteith, J.L., 1988. A Four-layer Model for the Heat Budget of. Homogeneous Land Surfaces. Quart. 7. Roy. Meteorol. Soc. 144, 373-398.

Daamen, C.C., 1993. Evaporation from Sandy Soils Beneath Crops in theSemi-arid Zone-a Study of the $U_{\text {se }}$ of Microlysimeters and Numerical Simulation. Thesis R6465, University of Reading, Reading, England.

Daamen, C.C., 1997. Two Source Model of Surface Fluxes for Millet Fields in Niger. Agric. For. Meteorol. 83, 205-230.

Franks, S.W., Beven, K.J., Quinn, P.F. and Wright, I.R., 1997. On the Sensitivity of Soil-Vegetation-Atmosphere Transfer (SVAT) Schemes: Equifinality and the Problem of Robust Calibration. Agric. For. Meteorol. 86, 63-75.

Gerstl, S.A.W. and Borel, C.C., 1992. Principles of the Radiosity Method Versus Radiative Transfer for Canopy Reflectance Modeling. IEEE Trans. Geosci. Remote Sensing 30, 271-275.

Goutorbe, J.P., Lebel, T., Dolman, A.J., Gash, J.H.C., Kabat, P., Kerr, Y.H.,Monteny, B., Prince, S.D., Stricker, J.N.M., Tinga, A. and Wallace, J.S., 1997. An Overview of HAPEX-Sahel: A Study in Climate and Desertification. 7. Hydrol. 189, 4-17.

Grant, I.P. and Hunt, G.E., 1969a. Discrete Space Theory of
Radiative Transfer I. Fundamentals. Proc. Roy. Soc. Lond. A 313, 183-198.

Grant, I.P. and Hunt, G.E., 1969b. Discrete Space Theory of Radiative Transfer II. Stability and Non-negativity, Proc. Roy. Soc. Lond. A 313, 199.

Henderson-Sellers, A., Yang, Z-L. and Dickinson, R.E., 1993. The project for Intercomparison of Land-Surface Parametrization Schemes. Bull. Am. Meteorol. Soc. 74, 1335-1349.

Hope, A.S., Petzold, D.E., Goward, S.N. and Ragan, R.M., 1986. Simulated Relationships Between Spectral Reflectance, Thermal Emissions and Evapotranspiration of a Soybean Canopy. Wat. Resour. Bull. 22, 1011-1019.

Monteith, J.L. and Unsworth, M.H., 1990. Principles of Environmental Physics. Edward Arnold, London, 2nd Edition.

Nicodemus, F.E., Richmond, J.C., Hsia, J.J, Ginsberg, I.W. and Limperis, T., 1977. Geometrical Considerations and Nomenclature for Reflectance. U.S. Department of Commerce, National Bureau of Standards, October 1977.

Pearson, D., 1997. Calculation of First-order Scattering in a Leafy Canopy by Reduction to Two Dimensions-II. Stochastic Optics. 7. Quant. Spectrose. Radiat. Transfer 57, 605-626.

Philip, J.R. and de Vries, D.A., 1957. Moisture Movement in Porous Materials under Temperature Gradients. Trans. Am. Geophys. Un. 38, 222-232.

Press, W.H., Teukolsky, S.A., Vetterling, W.T. and Flannery, B.P., 1992. Numerical Recipes in FORTRAN (Second Edition). Cambridge University Press.

Sellers, P.J., Mintz, Y., Sud, Y.C. and Dalcher, A., 1986. A Simple Biosphere Model (SiB) for Use within General Circulation Models. 7. Atmos. Sci. 43, 505-531.

Shuttleworth, W.J. and Wallace, J.S., 1985. Evaporation from Sparse Crops - an Energy Combination Theory. Quart. 7. Roy. Meteorol. Soc. 111, 839-855.

Taconet, O., Bernard, R. and Vidal-Madjar, D., 1986. Evapotranspiration over an Agricultural Region Using a Surface Flux/Temperature Model Based on NOAA-AVHRR Data. 7. Clim. Appl. Meteorol. 25, 284-307.

Verhoef, W., 1984. Light Scattering by Leaf Layers with Application to Canopy Reflectance Modelling-the SAIL Model. Remote Sensing Environ. 16, 125-141.

Wallace, J.S., Brouwer, J., Allen, S.J., Banthorpe, D., Blyth, E.M., Blyth, K., Bromley, J., Buerkert, A.C., Cantwell, M., Cooper, J.D., Cropley, F.D., Culf, A.D., Dolman, A.J., Dugdale, G., Gash, J.H.C., Harding, R.J., Harrison, R.G., Holwill, C.J., Jarvis, P.G., Levy, P.E., Lloyd, C.R., Malhi, Y.S., Massheder, J.M., Moncrieff, J.B., Pearson, D., Settle, J.J., Sewell, I.J., Sivakumar, M.V.K., Sudlow, J.D., Taylor, C.M. and Wilson, A.K., 1994. HAPEX-Sahel Southern SuperSite Report: An overview of the site and the experimental programme during the intensive observation period in 1992. Institute of Hydrology, Wallingford, UK. 55pp.

Wallace, J.S., Gash, J.H.C., McNeil, D.D. and Sivukamar, M.V.K., 1986. Measurement and Prediction of Actual Evaporation from Sparse Dryland Crops-Scientific Report on Phase 2 of ODA Project 149. ODA Report No. 149/3, Institute of Hydrology, Wallingford, UK.

Wallace, J.S., Gash, J.H.C. and Sivukamar, M.V.K., 1990. Preliminary Measurements of Net Radiation and Evaporation over Bare Soil and Fallow Bushland in the Sahel. Int. 7 . Climatology 10, 203-210. 
Wang, Y.P. and Jarvis, P.G., 1990. Description and Validation of an Array Model-MAESTRO. Agric. For. Meteorol. 51, 257-280.

Xue, Y., Sellers, P., Kinter, J.L. and Shukla, J., 1991. A Simplified Biosphere Model for Global Climate Studies. 7. Climate 4, 345-364.

\section{Table of symbols}

Here are listed and defined the main mathematical symbols used in the paper.

\section{$A_{L} \quad$ Leaf area index}

$a_{s} \quad$ Shortwave albedo of the soil

$a_{v} \quad$ Single scattering albedo of the vegetation

$\hat{B} \quad$ Defined in Eqn. 5

E A thermal irradiance

E A shortwave irradiance

$E_{3}(\cdot)$ An exponential integral

$M \quad$ A thermal exitance

M A shortwave exitance
$R_{n} \quad$ Net radiation

$R_{s} \quad$ Net radiation available to the soil

$R_{v} \quad$ Net radiation available to the vegetation

$\hat{R}_{s}$ : Reflection operator for isotropic shortwave radiation incident on the vegetation

$\hat{R}_{t} \quad$ Reflection operator for isotropic thermal radiation incident on the vegetation

$T_{a} \quad$ Temperature at the reference height

$T_{s} \quad$ Temperature of the surface of the soil

$T_{v} \quad$ Temperature of the vegetation

$\hat{T}_{s}$ Transmission operator for isotropic shortwave radiation incident on the vegetation

$\hat{T}_{t}$ Transmission operator for isotropic thermal radiation incident on the vegetation

$\alpha \quad$ Extinction coefficient or scattering efficiency

$\epsilon_{a} \quad$ Thermal emissivity of the sky or reference height

$\epsilon_{s} \quad$ Thermal emissivity of the soil

$\epsilon_{v} \quad$ Thermal emissivity of the vegetation

$\sigma \quad$ The Stefan-Boltzmann constant

$\tau \quad$ An optical depth 